\title{
A Cerebellar Model of Timing and Prediction in the Control of Reaching
}

\author{
Andrew G. Barto ${ }^{\dagger}$, Andrew H. Fagg ${ }^{\dagger}$, \\ Nathan Sitkoff ${ }^{\dagger}$, and James C. Houk* \\ ${ }^{\dagger}$ Department of Computer Science, University of Massachusetts \\ *Department of Physiology, Northwestern University Medical School
}

May 1998

\begin{abstract}
A simplified model of the cerebellum was developed to explore its potential for adaptive, predictive control based on delayed feedback information. An abstract representation of a single Purkinje cell with multistable properties was interfaced, via a formalized premotor network, with a simulated single degree-of-freedom limb. The limb actuator was a nonlinear spring-mass system based on the nonlinear velocity dependence of the stretch reflex. By including realistic mossy fiber signals, as well as realistic conduction delays in afferent and efferent pathways, the model allowed the investigation of timing and predictive processes relevant to cerebellar involvement in the control of movement. The model regulates movement by learning to react in an anticipatory fashion to sensory feedback. Learning depends on training information generated from corrective movements and uses a temporally-asymmetric form of plasticity for the parallel fiber synapses on Purkinje cells.
\end{abstract}




\section{Introduction}

The neural commands that control rapid limb movements appear to be comprised of pulse components followed by smaller step components (Ghez 1979; Ghez and Martin 1982), analogous to the pulse-step commands that control rapid eye movements (Robinson 1975). In the case of eye movements, the pulse component serves to overcome the internal viscosity of the muscles, thus moving the eye rapidly to the target, whereupon the step component holds the eye at its final position. Limb movements involve more inertia than eye movements, so the pulse activation of the agonist muscle must end part way through the movement, and a braking pulse in the antagonist muscle is needed to decelerate the mass of the limb. Ghez and Martin (1982) showed that the braking pulse is produced by a stretch reflex in the antagonist muscle. The central control problem, therefore, is to terminate the pulse phase of the command sent to the agonist muscle at an appropriate time during the movement. The dynamics of the stretch reflex should then bring the movement to a halt at a desired endpoint. Since the pulse must terminate well in advance of the achievement of the desired endpoint, this is a problem of timing and prediction in control. In this article we present a model of how the cerebellum may contribute to the predictive control of limb movements.

The model is a simplified version of the adjustable pattern generator (APG) model being developed by Houk and colleagues (Berthier et al. 1993; Houk et al. 1990; Sinkjær et al. 1990) to test the computational competence of a conceptual framework for understanding the brain mechanisms of motor control (Houk 1989; Houk and Barto 1992; Houk et al. 1993; Houk and Wise 1995; Houk et al. 1996). The model has a modular architecture in which single modules generate elemental motor commands with adjustable time courses, and multiple modules cooperatively produce more complex commands. The APG model is constrained by the modular anatomy of the cerebellar cortex and its connections with the limb premotor network, by the physiology of the neurons comprising this network, and by properties of cerebellar Purkinje cells (PCs). However, it is purposefully abstract to allow us to explore control and learning issues in a computationally feasible manner. The model presented here corresponds to a single module of the APG model consisting of a single unit representing a PC. This unit is modeled as a collection of nonlinear switching elements, which we call dendritic zones, representing segments of a $\mathrm{PC}$ dendritic tree.

Our previous modeling studies dealt mainly with two issues: (1) demonstration that a single module can learn to generate appropriate one-dimensional, 
variable-duration velocity commands (Houk et al. 1990) and (2) a preliminary demonstration that an array of 48 modules can learn to function cooperatively in the control of a simulated non-dynamic, two-joint planar limb (Berthier et al. 1993). In these previous simulations, the input layer of the cerebellum, the representation of PCs, and the complexity of the learning problem were greatly simplified. In the present article, we employ a more realistic input representation based on what is known about movement-related mossy fiber (MF) signals in the intermediate cerebellum of the monkey (Van Kan et al. 1993a) and the Marr-Albus architecture of the granular layer (Tyrrell and Willshaw 1992). In addition, we use a more complex dynamic spring-mass system (although it is still one-dimensional), and we include realistic conduction delays in the relevant signal pathways. The model also makes use of a trace mechanism in its learning rule. Preliminary results appear in Buckingham et al. (1995) and Barto et al. (1996).

We first describe the nonlinear spring-mass system and discuss some of its properties from a control point of view. The following section presents the details of the model. We then present simulation results demonstrating the learning and control abilities of a single dendritic zone followed by similar results for a model with multiple dendritic zones. We conclude with a discussion of these results.

\section{Pulse-Step Control of a Nonlinear Plant}

The limb motor plant has prominent nonlinearities that have a strong influence on movement and its control. The plant model used in this study is a spring-mass system with a form of nonlinear damping based on studies of human wrist movement (Gielen and Houk 1984; Wu et al. 1990):

$$
M \ddot{x}+B(\dot{x})^{\frac{1}{5}}+K\left(x-x_{e q}\right)=0,
$$

where $x$ is the position (in meters) of an object of mass $M(\mathrm{~kg})$ attached to the spring, $x_{e q}$ is the resting, or equilibrium, position, $B$ is the damping coefficient, and $K$ is the spring stiffness (Fig. 1A). This fractional-power form of nonlinear damping is derived from a combination of nonlinear muscle properties and spinal reflex mechanisms, the latter driven mainly by feedback from muscle spindle receptors (Gielen and Houk 1987). Setting $M=1$, $B=3$, and $K=30$ produces trajectories that are qualitatively similar to those observed in human wrist movement (Wu et al. 1990).

Nonlinear damping of this kind enables fast movements that terminate with little oscillation. Fig. 1B is a graph of the damping force as a function of 


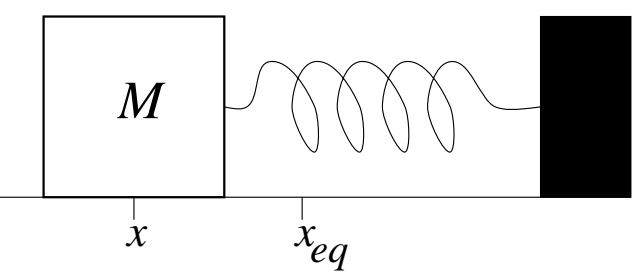

A
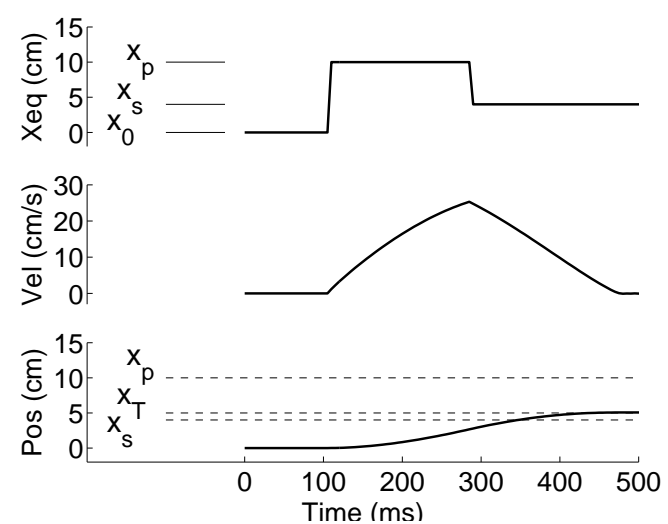

$\mathrm{C}$

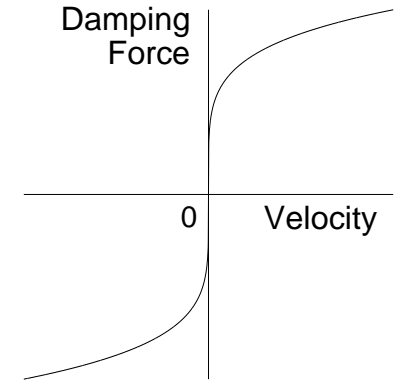

$\mathrm{B}$

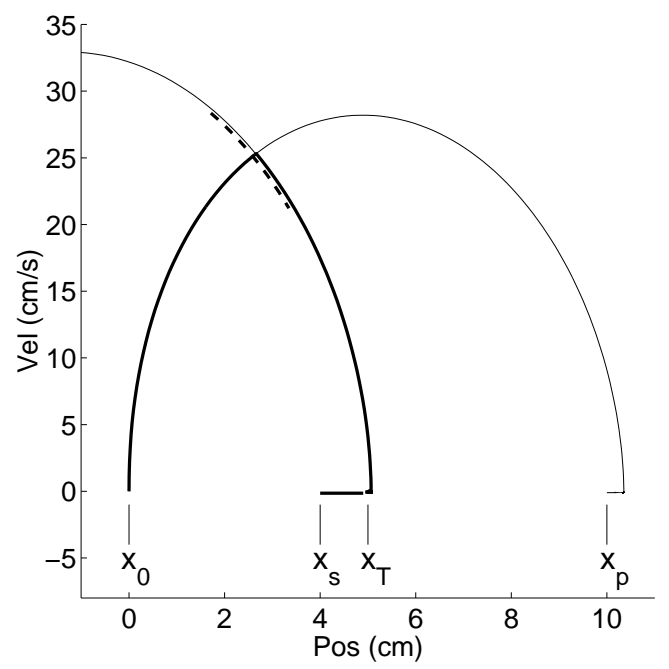

$\mathrm{D}$

Figure 1: Pulse-Step Control of a Simplified Motor Plant. Panel A: SpringMass System. $M$, mass; $x$, position; $x_{e q}$, resting, or equilibrium, position. Panel B: Nonlinear Damping Force as a Function of Velocity. The plant's effective damping coefficient (the graph's slope) increases rapidly as the velocity magnitude decreases to zero. Panel C: Pulse-Step Control. Control of a movement from initial position $x_{0}=0$ to target endpoint $x_{T}=5 \mathrm{~cm}$. Top-The pulse-step command. Middle-Velocity as a function of time. Bottom-Position as a function of time. Panel D: Phase-Plane Trajectory. The bold line is the phase-plane trajectory of the movement of Panel C. The dashed line is a plot of the states of the spring-mass system at which the command should switch from pulse to step so that the mass will stick at the endpoint $x_{T}=5 \mathrm{~cm}$ starting from a variety of different initial states. 
velocity. As velocity decreases, the effective damping coefficient (the curve's slope) increases radically when the velocity gets sufficiently close to zero. This causes a decelerating mass generally to "stick" at a non-equilibrium position, thereafter drifting extremely slowly toward $x_{e q}$. We call the position at which the mass sticks (defined here as the position at which the absolute value of its velocity falls and remains below $0.9 \mathrm{~cm} / \mathrm{sec}$ ) the endpoint of a movement, denoted $x_{e}$. For all practical purposes, this is where the movement stops.

The control signal in our model sets the equilibrium value $x_{e q}$, which represents a central motor command setting the threshold of the stretch reflex (Feldman 1966; Houk and Rymer 1981). Pulse-step control is effective in producing rapid and well-controlled positioning of the mass in this system. As shown in Fig. 1C, the control signal switches from a pulse level $x_{e q}=x_{p}$, to a smaller step level $x_{e q}=x_{s}$. Also shown are the time courses of the velocity and position (middle and bottom) for the resulting movement. Inserting a low-pass filter in the command pathway, a common feature of muscle models, would produce velocity profiles more closely matching those of actual movements, but we have not been concerned with this issue.

Fig. 1D shows the phase-plane trajectory (velocity plotted against position) followed by the state of the spring-mass system during pulse-step control. When the pulse is being applied, the state follows a trajectory that would end at the equilibrium position $x_{p}=10 \mathrm{~cm}$ if the pulse were to continue. When the step begins, the state switches to the trajectory that ends at the equilibrium position $x_{s}=4 \mathrm{~cm}$, but the mass sticks at the target endpoint $x_{T}=5 \mathrm{~cm}$ before reaching this equilibrium position. Thus, simply setting the equilibrium position to the target endpoint as suggested by the equilibriumpoint hypothesis (Bizzi et al. 1992; Feldman 1966, 1974) is not a practical solution to the endpoint positioning task for this system. The dashed line in Fig. 1D is an approximate plot of the states at which the switch from pulse to step should occur so that movements starting from a variety of initial states will stick at $x_{T}=5 \mathrm{~cm}$. This switching curve has to vary as a function of the target endpoint. If the switch from pulse to step occurs too soon (late), the mass will undershoot (overshoot) $x_{T}$.

In developing a model of pulse-step control of the limb, one can profit from analogies, where appropriate, with the extensive literature on pulse-step control of saccadic eye movements. However, an important difference between eye and limb control is the absence of a stretch reflex for regulating primate eye muscle activity (Keller and Robinson 1971). As a consequence, models of the eye motor plant do not contain the nonlinear damping mechanism present in Eq. 1. As mentioned above, the stretch reflex is important in generating a 
braking pulse in the antagonist muscles needed to decelerate the limb (Ghez and Martin 1982). In fact, the stretch reflex is the predominant mechanism responsible for the entire decelerating portion of the trajectory in Fig. 1D. The stretch reflex is also the main mechanism causing the limb to stick at a non-equilibrium position, as witnessed by the drift in limb position that occurs in deafferented patients that lack a stretch reflex (Ghez et al. 1990). For eye movements, the prevention of post-saccadic drift is critically dependent on the precise regulation of the step component of the pulse-step command (Optican and Robinson 1980). Although it is likely that the step component is also regulated for limb movements, relatively little is known about this mechanism. For the purposes of the present paper, we assume the presence of a fixed step component and rely on nonlinear damping for causing the limb to stick at an endpoint.

\section{Model Architecture}

Both limb and saccadic control systems are highly distributed, involving the cerebral cortex, basal ganglia, cerebellum, tectum, brainstem and spinal cord. The focus here is on the special role of the cerebellum, which exerts its influence on movement by way of premotor networks. For both limb movements and saccades, there are two levels of premotor network. The upper level is the cortico-rubro-cerebellar network for the limb (Houk et al. 1993) and the tecto-reticulo-cerebellar network for saccades (Houk, et al. 1992; Arai et al. 1994). These upper-level networks feed control signals to a lower level comprised of a propriospinal network for the limb (Alstermark et al. 1987a) and a brainstem burst network for saccades (Robinson, 1975). Since the emphasis in this paper is on the cerebellar cortex, the premotor networks will be given only a formal representation. We assume that the propriospinal network, in analogy with the brainstem burst network, can only generate relatively crude commands that typically produce dysmetric

movements when it operates on its own. However, the system is capable of orthometric control when the cerebellum and upper premotor networks are operative. In order to focus on the critical control functions of PCs in the cerebellar cortex, we will represent the cortico-rubro-cerebellar network as simply an inverting mechanism that converts the inhibitory output of PCs into a positive command signal. For simplicity, we further assume that the output of this cortico-rubro-cerebellar network acts directly on spinal output rather than functioning through the propriospinal network.

The model's main component is a single unit representing a cerebellar PC, whose input is derived from a sparse, expansive encoding of mossy fiber 


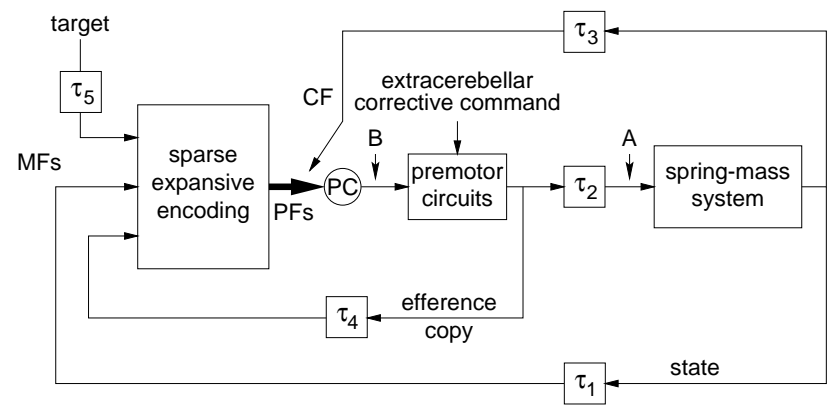

A

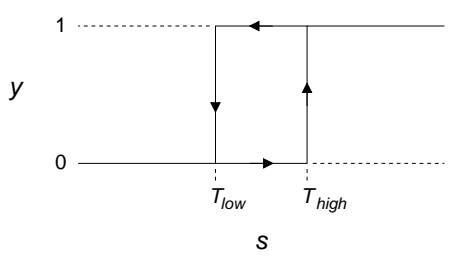

$\mathrm{B}$

Figure 2: Model Architecture. Panel A: Block Diagram. PC, Purkinje cell; MFs, mossy fibers; PFs, parallel fibers; CF, climbing fiber; $\tau_{i}, i=1, \ldots, 5$, conduction delays. The labels A and B mark places in the feedback loop to which we refer in discussing the model's behavior. Panel B: Dendritic Zone Hysteresis. DZ activation, $y$, switches from 0 to 1 when the input weighted sum, $s$, exceeds threshold $T_{\text {high }}$, and switches from 1 to 0 when $s$ drops below $T_{\text {low }}$.

(MF) signals (Fig. 2A). In defining how the MFs encode information about the spring-mass system, we followed what is known about movement-related MF signals in the intermediate cerebellum of the monkey, where MFs exhibit discharge patterns involving diverse combinations of tonic and phasic components, as well as a variety of onset times relative to the time of movement onset (Van Kan et al. 1993a).

To represent this diversity, the model has a total of 2000 MFs, 800 of which encode information about single variables $x, \dot{x}, x_{e q}$, or $x_{T}$ (200 MFs devoted to each), with the remaining $1200 \mathrm{MFs}$ encoding information about pairwise combinations of these variables. Each of the MFs representing a single variable uses a saturated ramp encoding. For example, as the mass's position increases, the firing rate of a pure position-related MF remains zero until a threshold is exceeded, and increases linearly thereafter until saturating at a maximum firing rate. The thresholds are distributed uniformly over the relevant variable ranges, and several slopes and saturation levels are used. ${ }^{1}$ In addition, the signal conveyed by each pure position and velocity MF

\footnotetext{
${ }^{1}$ Thresholds are distributed at uniform intervals over the ranges of the relevant variables $\left([-0.5,7.5] \mathrm{cm}\right.$ for $x ;[-25,25] \mathrm{cm} / \mathrm{sec}$ for $\dot{x} ;[0,1]$ for $x_{e q}$; and $[3,7] \mathrm{cm}$ for $\left.x_{T}\right)$. The slopes were set so that the ramp covers $50 \%, 25 \%$, or $12.5 \%$ of the variable's range. Half of the slopes are negative, so that the MF decreases in activity as its coded variable increases. Saturation levels differ slightly as a function of threshold, with higher thresholds being associated with higher saturation levels. This roughly normalizes the average activity level of the MFs.
} 
is delayed relative to spring-mass movement by an amount chosen uniformly at random from between 15 and $100 \mathrm{~ms}$ ( $\tau_{1}$ in Fig. 2A). The delay ranges for this and the following types of MFs are within those observed for the intermediate cerebellum of the monkey (Van Kan et al. 1993a). The signals of the efference copy MFs (representing $x_{e q}$ ) are delayed between 40 and 150 $\mathrm{ms}$ (uniform random) relative to the motor command ( $\tau_{4}$ in Fig. $\left.2 \mathrm{~A}\right)$. The signal of each target position MF (representing $x_{T}$ ) is delayed between 0 and $100 \mathrm{~ms}$ (uniform random) from the start of a trial ( $\tau_{5}$ in Fig. 2A). The signal conveyed by each of the $1200 \mathrm{MFs}$ representing pair-wise combinations of the single variables is a weighted sum of the signals of two single-variable MFs: 400 are combinations of pure $x$ and $\dot{x}$ MFs, 400 are combinations of pure $x$ and $x_{e q}$ MFs, and 400 are combinations of pure $x_{T}$ and $\dot{x}$ MFs. Within these classes, the pairs of MFs were chosen uniformly at random, and the weights, which are positive and sum to one for each MF, were selected uniformly at random. The relative number of MFs in these various classes is consistent with the proportions observed by Van Kan et al. (1993a). The total number of MFs was chosen for computational reasons: we wanted to ensure that the model could accurately represent the transformation required by the control task. We did not rule out the possibility that fewer MFs might also suffice.

We set the efferent delay from the $\mathrm{PC}$ to the spring-mass system via premotor circuits to $100 \mathrm{~ms}\left(\tau_{2}\right.$ in Fig. 2A), which is within the range observed for this pathway in the intermediate cerebellum of the monkey (Van Kan et al. 1993b), although we experimented with other values as well (Sec. 5.1). With this delay, the MF delay ranges described above imply that the onset of movement-related discharge of the MFs that use efference copy information can lead movement onset by as much as $60 \mathrm{~ms}$, or lag it by as much as 50 ms. On the other hand, movement-related discharge of MFs relying only on proprioceptive information always lags movement by between 15 and $100 \mathrm{~ms}$.

Patterns of MF activity are recoded to form sparse activity patterns over 40, 000 binary parallel fibers (PFs) which synapse on the PC. This form of $\mathrm{PF}$ state encoding is similar to that used in numerous models of the cerebellum, such as those of Marr (1969) and Albus (1971). We selected this number of PFs to ensure that the model could realize the required transformation. With as few as 30,000 PFs, learning progresses at a slower rate and asymptotes at a higher average endpoint error. However, with 60,000 PFs, an improvement in learning performance is not observed. Each PF is the output of a granule unit that sums excitatory input from 4 randomly chosen MFs. We assumed that local competition takes place among granule units, allowing only 80 of the units to fire (output $=1$ ) at the same time. Marr (1969) and Albus (1971) hypothesized that this competition arises from inhibitory interactions through Golgi cells. We implemented this competition 
by dividing the granule cell population into 80 Golgi-cell receptive fields, each comprising 500 granule units, and allowing only the most active unit in each field to fire at any time step of the simulation (although the model does not explicitly contain units representing Golgi cells). Thus, at each time step, the $\mathrm{PF}$ input to the $\mathrm{PC}$ is a pattern of 40,000 binary values containing 80 ones.

The PC in the model consists of a number of dendritic zones (DZs) representing segments of the dendritic tree. Our representation of DZs is motivated by observations of plateau potentials in PC dendrites (Llinás and Sugimori 1980; Ekerot and Oscarsson 1981; Campbell et al. 1983; Andersson et al. 1984). These long-lasting potentials (up to several hundred $\mathrm{ms}$ in duration) represent a form of bistability, which results from hysteresis produced by the dendritic ion-channel system. A number of researchers have suggested that dendritic or neuronal bistability resulting from hysteresis can be computationally useful (Hoffman 1986; Benson et al. 1987; Kiehn 1991; Houk et al. 1990; Wang and Ross 1990; Gutman 1991, 1994), and Yuen et al. (1995) showed how these properties can arise in a biophysical model of the $\mathrm{PC}$ dendrite.

Each DZ in the model is a linear threshold unit with hysteresis. Let $s(t)=$ $\sum_{i} w_{i}(t) \phi_{i}(t)$, where $\phi_{i}(t)$ denotes the activity of PF $i$ at time $t$ and $w_{i}(t)$ is the efficacy, or weight, at time step $t$ of the synapse by which PF $i$ influences the PC dendritic segment comprising the DZ. The activity of the DZ at time $t$, denoted $y(t)$, is either 1 or 0 , respectively representing a state of high or low activity. DZ activity depends on two thresholds: $T_{\text {low }}$ and $T_{\text {high }}$, where $T_{\text {low }}<T_{\text {high }}$. The activity state switches from 0 to 1 when $s(t)>T_{\text {high }}$, and it switches from 1 to 0 when $s(t)<T_{\text {low }}$ (Fig. 2B). If $T_{\text {high }}=T_{\text {low }}$, the DZ is the usual linear threshold unit. Unlike plateau potentials, which tend to reset spontaneously after a few hundred milliseconds (Llinás and Sugimori 1980; Campbell et al. 1983; Andersson et al. 1984), the state of a DZ remains constant until actively switched by input. We have not yet explored the consequences of spontaneous resetting in our model. In the simulations reported below, we investigated the effects of several settings of $T_{\text {high }}$ and $T_{\text {low }}$.

The PC's overall activity level at any time is equal to the fraction, $f$, of its DZs that are in state 1 at that time. In a more detailed model, the PC would inhibit nuclear cells, thereby regulating the buildup of activity in cortico-rubro-cerebellar loops from which motor commands are derived (Houk et al. 1993). The simpler model described here does not include an explicit representation of these premotor circuits. The motor command, $x_{e q}$, is simply defined to be $4 f+10(1-f)$, which means that when the $\mathrm{PC}$ is 
maximally active $(f=1)$, the equilibrium position is the "near" position of $4 \mathrm{~cm}$, and when it is minimally active $(f=0)$, the equilibrium position is the "far" position of $10 \mathrm{~cm}$. These values determine the range of target endpoints for which the model is able learn accurate positioning commands, but the model is not otherwise sensitive to the specific values. This definition of the motor command reflects the inhibitory effect the $\mathrm{PC}$ would have on cortico-rubro-cerebellar loops. As a result, pauses in the PC's activity would disinhibit activity of premotor circuits which activate an agonist muscle for rightward movement.

We studied three versions of the model that differ in the number of DZs and how the PF input is distributed among them. In the simplest version, a single DZ receiving input from all 40,000 PFs. In the other versions, the $\mathrm{PC}$ consists of $8 \mathrm{DZs}$. In one of these, each DZ receives input from all of the PFs; in the other, each DZ receives input from a separate subfield of 5,000 PFs. The latter version of the model, which is more realistic due to the orthogonal relationship between PFs and the flattened dendritic trees of PCs in the cerebellum, learned somewhat slower than the other 8-DZ model, but its behavior was similar in other respects (Sec. 5.2).

\section{Learning}

All the DZs comprising the $\mathrm{PC}$ in the model receive training information from a signal representing discharge of a climbing fiber (CF). This signal provides information about the spring-mass system with a delay of $20 \mathrm{~ms}$ ( $\tau_{3}$ in Fig. 2A), which is within the physiological range for CF signals in cats (Gellman et al. 1983). The nature of the training information supplied by the model's CF is an extrapolation of what is known about the responsiveness of proprioceptive CFs, which respond to particular directions of limb movement and appear to signal "unexpected" passive movements, being suppressed during active (hence expected) movements (Gellman et al. 1985). We hypothesize that by monitoring the proprioceptive consequences of corrective movements generated by other structures, modules of the cerebellum can learn to regulate motor commands so that they produce more efficient and accurate movement. We follow Berthier et al. (1993) in assuming that the propriospinal premotor network generates simple corrective movements when a movement is inaccurate. These corrective movements do not have to be particularly accurate themselves; they only need to reduce the endpoint error. The literature upon which these assumptions are based is reviewed in some detail in an earlier paper (Berthier et al., 1993; see Sec. 6). Although 
a sequence of such corrective movements alone can produce small final endpoint error, the sequence would be slow and dynamically erratic. The role of the cerebellum, we hypothesize, is to eliminate the need for corrective movements by learning to suitably regulate the initial movement.

In the model, whenever the mass is coming to rest at a point not near the target endpoint, an extracerebellar motor command in the form of a single rectangular pulse is generated, causing movement in the correct direction. ${ }^{2}$ In response to each rightward corrective movement, the model's directionallysensitive CF produces a single discharge. The CF is silent during leftward corrective movements (although the $\mathrm{CF}$ to a module activating a muscle for leftward movement, if one were present in the model, would discharge in this case). This follows a key assumption that the responsiveness of a PC's $\mathrm{CF}$ to movement in a given direction is matched to the degree to which that PC's module is capable of contributing to movement in that direction (see Berthier et al., 1993, for additional details). We also assume a low background firing rate for the $\mathrm{CF}$ in the absence of corrective movements. Letting $c(t)$ denote $\mathrm{CF}$ activity at time step $t$, the model implements these assumptions by setting $c(t)=1$ at the initiation of each rightward corrective movement, $c(t)=0$ for the remainder of the rightward movement, $c(t)=0$ during leftward corrective movements, and otherwise $c(t)=\beta=0.025$, which represents a low background firing rate. ${ }^{3}$

As a result of a corrective movement, the weights of each DZ should change so that the $\mathrm{PC}$ contributes to a more accurate motor command. In response to a rightward corrective movement, the weights should change so as to increase the duration of the pulse phase of the command (since the movement stopped short of the target endpoint), and in response to a leftward corrective movement, the weights should change so as to decrease the duration of the pulse phase of the command (since the movement overshot the target endpoint). Accomplishing this with a simple learning rule is difficult because the training information in the form of $\mathrm{CF}$ activity is significantly delayed with respect to the relevant DZ activity due to the combined effects of movement duration and conduction latencies. To learn under these conditions, the model adopts Klopf's $(1972,1982)$ hypothesis of synaptic "eligibility traces." Appropriate

\footnotetext{
${ }^{2}$ Whenever the mass has been "stuck" for $150 \mathrm{~ms}$ more than $0.1 \mathrm{~cm}$ from the target endpoint, the motor command, $x_{e q}$, is set to a value that causes movement toward the target. Specifically, $x_{e q}=x_{T}+a$ for undershoot, and $x_{e q}=x_{T}-a$ for overshoot, where $a>0$ was chosen to be sufficiently large to overcome the high low-velocity viscosity. Here, we used $a=5 \mathrm{~cm}$.

${ }^{3}$ We experimented with a more realistic representation of background activity in which $c(t)=1$ with probability $\beta$ for each background time step $t$. The results were essentially the same, except that the learning process required about 2.5 times as many trials.
} 
activity at a synapse is hypothesized to set up a synaptically-local memory trace that makes the synapse "eligible" for modification if, and when, the appropriate training information arrives within a short time period. This allows the learning rule to modify synaptic weights based on synaptic actions that occurred prior to the availability of the relevant $\mathrm{CF}$ training information. An example eligibility trace for one PF-to-PC synapse is shown in the bottom plot of Fig. 4A. This eligibility trace spans the interval from the time of the presynaptic PF's activity until later CF discharges occur (plot CF in the figure), at which times this synapse's weight is modified.

To define the learning rule, we have to specify how synapses become eligible for modification and how CF activity alters the synaptic weights based on the eligibility of synapses. We first describe the eligibility process. The idea is that a synapse becomes eligible for modification if its presynaptic PF was active in the recent past at the same time that the synapse's DZ was in state 1. Eligibility then persists as a graded quantity - a trace - that reflects both how frequently and how long in the past this eligibility-triggering condition was satisfied for that synapse. Although learning is not sensitive to the exact time course of eligibility traces, a synapse should reach peak eligibility at roughly the time at which a relevant $\mathrm{CF}$ discharge would reach the $\mathrm{PC}$. By a relevant $\mathrm{CF}$ discharge, we mean one produced by a correction to a movement that was influenced by the eligibility-triggering activity at the given synapse.

One of the simplest methods for computing eligibility is to simulate a secondorder linear filter whose input is 1 whenever the triggering condition is satisfied, and 0 otherwise. The filter's parameters were set so that its impulse response rises to a peak about $255 \mathrm{~ms}$ after the triggering event, and then decays asymptotically to zero with a time constant of approximately $600 \mathrm{~ms}$. A synapse is therefore maximally eligible $255 \mathrm{~ms}$ after the triggering event and becomes effectively ineligible approximately 2 sec later, assuming no additional triggering events occur (see the bottom plot of Fig. 4A). This time course is appropriate for the movement durations and conduction delays in the present model. An intracellular signal transduction mechanism for producing this kind of eligibility trace was proposed in Kettner et al. (1997). We also found it useful to limit the magnitude of eligibility so that prolonged periods during which the triggering condition is satisfied do not lead to excessively high eligibility, and hence to large weight changes. In the discussion we comment on the biological realism of the eligibility idea.

Letting $e_{i}(t)$ denote the eligibility of synapse $i$ at time $t$, the model generates the eligibility trace for each synapse $i$ by the following difference equations 
involving the intermediate variables $\bar{e}_{i}$ and $\hat{e}_{i}$ :

$$
\begin{aligned}
& \bar{e}_{i}(t)=.98 \bar{e}_{i}(t-1)+.02 y(t) \phi_{i}(t), \\
& \hat{e}_{i}(t)=.98 \hat{e}_{i}(t-1)+.02 \bar{e}_{i}(t-1), \\
& e_{i}(t)=\min \left\{\hat{e}_{i}(t), 0.1\right\}
\end{aligned}
$$

where $y(t)$ is the binary activity state of the synapse's DZ at time step $t$, and $\phi_{i}(t)$ is the activity of the presynaptic PF. Each time step in the model represents $5 \mathrm{~ms}$ of real time.

The remainder of the model's learning mechanism is a rule determining how the weights of eligible synapses are altered by CF activity. The logic of this learning rule is a result of the following reasoning. When the weights of a DZ's eligible synapses decrease, that DZ becomes less likely to switch to state 1 in the future when a situation (represented by a pattern of PF activity) is encountered that is similar to the one that was present when the eligibility trace was initiated. This tends to prolong the pulse phase of the motor command by delaying the DZ's contribution to PC inhibition, which increases movement duration and moves the initial movement's endpoint to the right. Thus, the weights of eligible synapses should decrease as a result of each rightward corrective movement. Since the CF produces a discharge on each rightward corrective movement, CF discharge should cause depression of the eligible synapses. On the other hand, increasing the weights of a DZ's eligible synapses makes that DZ more likely to switch to state 1 under similar circumstances in the future, which tends to shorten the pulse phase of the motor command, thus decreasing movement duration and moving the endpoint leftward. Therefore, leftward corrective movements should cause potentiation of the eligible synapses. In the model this is accomplished by letting the CF signal drop below its background rate during leftward corrective movements.

The following learning rule implements this logic:

$$
\begin{aligned}
\Delta w_{i}(t) & =-\alpha e_{i}(t)\left[c\left(t-\tau_{3}\right)-\beta\right], \\
w_{i}(t) & =\max \left\{w_{i}(t-1)+\Delta w_{i}(t), 0\right\},
\end{aligned}
$$

where $\alpha>0$ is a parameter influencing the rate of learning which was set to $2 \times 10^{-3}$ in the simulations described below. ${ }^{4}$ The term $\beta$ implies that weights do not change during background CF activity, and that eligible weights increase during leftward corrective movements when CF activity drops below

\footnotetext{
${ }^{4}$ This was chosen to be such a small value because the resultant change in PC activation due to each learning step could be 80 times larger since 80 of the PF inputs are 1 at each time step.
} 
its background rate. Note that since $\beta \ll 1$, weight increases are much smaller than weight decreases. The term $c\left(t-\tau_{3}\right)$, where $\tau_{3}=20 \mathrm{~ms}$ is the CF conduction delay, is the CF signal that reaches the synapse at time $t$. Since eligibility, $e_{i}(t)$, is a multiplicative factor, weights change in proportion to their degree of eligibility. All the DZs comprising the model's PC learn independently according to this rule.

To summarize the model's learning mechanism, training information is supplied by $\mathrm{CF}$ responses to corrective movements. The CF for the single module described here discharges reliably in response to rightward corrective movements. This follows from the specificity of the CF system and the assumption that this module controls an agonist for rightward movement. Rightward corrective movements therefore raise the CF's activity above its background rate. For leftward corrective movements, the CF's activity decreases slightly below its background rate. The weights of the synapses from PFs to the DZs comprising the model's PC change in response to CF activity so that the duration of the pulse phase of the motor command is increased in the case of rightward corrective movements and decreased in the case of leftward corrective movements. The model uses eligibility traces to bridge the time interval between the activity of the DZs and the relevant later CF activity. A synapse becomes eligible for modification when presynaptic activity coincides with the postsynaptic DZ being in state 1. Eligibility is realized as a synaptically-local trace that persists for several seconds after the coincidence of pre- and postsynaptic activity. When CF activity rises above its background level, the weights of the synapses are depressed in proportion to their current degree of eligibility, which tends to lengthen the pulse phase of the command. When CF activity falls below its background level, synapses are facilitated in proportion to their eligibility, which tends to shorten the pulse phase of the command.

\section{Simulations}

\subsection{Single Dendritic Zone}

We performed a number of simulations of a single DZ learning to control the nonlinear spring-mass system. We trained the DZ to move the mass from initial positions selected randomly from the interval $[0,2 \mathrm{~cm}]$ to a target position randomly set to 3,4 , or $5 \mathrm{~cm}$. DZ state 0 corresponded to the pulse phase of a motor command, which set a "far" equilibrium position of $10 \mathrm{~cm}$; DZ state 1 corresponded to the step phase, which set a "near" 


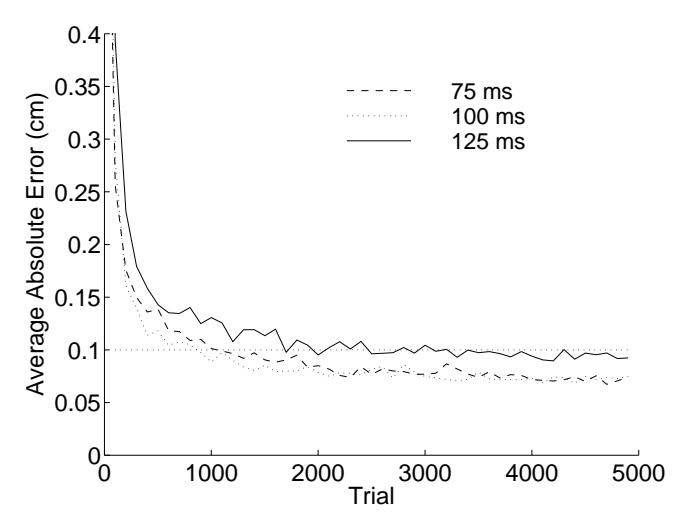

A

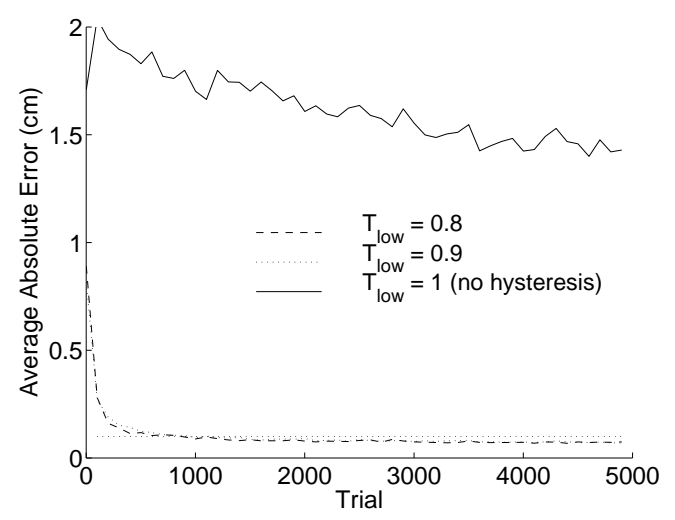

$\mathrm{B}$

Figure 3: Endpoint Error $\left(\left|x_{e}-x_{T}\right|\right)$ as a Function of Trial Number for Single DZ Learning. Each plotted point is an average over a bin of 50 trials of 10 learning runs. The dotted horizontal line shows the minimum threshold above which corrective movements were generated. Panel A: Effect of Loop Delay. Plots for efferent delays $\left(\tau_{2}\right)$ of 75,100 , and $125 \mathrm{~ms}$. Here, $T_{\text {low }}=0.8$ and $T_{\text {high }}=1$. Panel B: Effect of Hysteresis. Plots for $T_{\text {high }}=1$ and $T_{\text {low }}=.8$, .9 , and 1 (no hysteresis). Efferent delay $\left(\tau_{2}\right)$ was $100 \mathrm{~ms}$.

equilibrium position of $4 \mathrm{~cm}$ (see Sec. 2). Each simulation consisted of a series of trial movements. At the beginning of the first trial movement, we randomly initialized all 40,000 weights so that the weighted sum, s, fell uniformly between 0.68 and 1.48 for any initial pattern of PF activity. Each trial began when the state of the DZ was set to 0 . We initialized each eligibility trace, $e_{i}(t)$, to $0\left(\bar{e}_{i}(t)\right.$ and $\hat{e}_{i}(t)$ were also set to 0$)$. We also set the pattern of MF activity to be consistent with the initial state of the springmass system.

To study the influence of loop delay on learning and performance, we conducted simulations in which the loop delay was varied by setting the efferent delay $\left(\tau_{2}\right.$ in Fig. $2 \mathrm{~A}$ ) to 75,100 , or $125 \mathrm{~ms}$. Fig. 3A shows how the endpoint error decreased with trials for the various efferent delays (with $T_{\text {low }}=0.8$ and $\left.T_{\text {high }}=1\right)$. The DZ's behavior is largely insensitive to this range of delays. In each case, the average absolute error rapidly dropped below $0.1 \mathrm{~cm}$ (dotted line in Fig. 3A), the trigger criterion for the extracerebellar corrective movement. In all the simulations reported below, we set $\tau_{2}=100 \mathrm{~ms}$.

However, the model's behavior is sensitive to the amount of DZ hysteresis. Fig. 3B shows how endpoint error decreased over trials for several different values of $T_{\text {low }}$, with $T_{\text {high }}$ fixed at 1 . Learning was seriously disrupted when 
there was no hysteresis $\left(T_{\text {low }}=T_{\text {high }}=1\right)$. In all simulations reported below, $T_{\text {low }}=0.8$ and $T_{\text {high }}=1$, unless noted otherwise.

Figure 4 shows time courses of relevant variables at different stages in learning to move to target endpoint $x_{T}=5 \mathrm{~cm}$ from initial position $x_{0}=0$. Early in learning (4 trials, Panel A), the DZ switched back to state 1 too soon (plot $f$ ), which caused the mass to undershoot the target. Because of this undershoot, the extracerebellar system (EC) generated a corrective movement. In fact, a sequence of six corrective movements was generated because all but the last failed to bring the mass close enough to the target. Each corrective movement caused a CF discharge. The resulting movement accurately reached the target but along a slow and irregular trajectory.

Plotted at the bottom of Fig. 4 is the binary activity of an arbitrarily selected PF and the eligibility trace of its synapse onto the PC. Note that each discharge of the PF contributed to the trace because the DZ was in state 1 at these times. The weight of this PF's synapse (not shown) decreased when the $\mathrm{CF}$ discharge coincided with nonzero eligibility. The decrease of this weight, along with decreases of many others, tended to prolong the pulse-phase of the motor command by delaying the DZ's switch to state 1 . None of the synaptic weights increased during this trial because there was no leftward corrective movement (see Fig. 7A for an example of a trial with a leftward corrective movement). Later in learning (after 1,000 trials, Panel B), the model consistently produced accurate reaching with fast, smooth movements requiring no corrections (and hence causing no CF discharges). Note that to accomplish this, the DZ learned to switch to state 1 well before (about 300 $\mathrm{ms}$ ) the endpoint was reached.

Fig. 5A shows the paths of a number of movements controlled by a welltrained DZ. The initial position of the mass for each movement is indicated by the circle at the left end of each line, and the target endpoints are indicated by the vertical dashed lines. The asterisk on each path marks the position of the mass when the DZ switched state from 0 to 1 . The endpoint of each movement is indicated by the ' $x$ ' at the right end of each line. One can see that the movements were accurate across a range of initial positions and target endpoints. It is apparent that the DZ switched state well before the end of each movement.

Fig. 5B shows two representations (dashed lines labeled A and B) of the switching curve learned by the DZ for target $x_{T}=5 \mathrm{~cm}$, together with three sample phase-plane trajectories. Switching curve A is the switching curve as it appears after the efferent delay $\tau_{2}$, that is, as seen from the point marked A in Fig. 2A. When the spring-mass system's state crosses this curve, the 


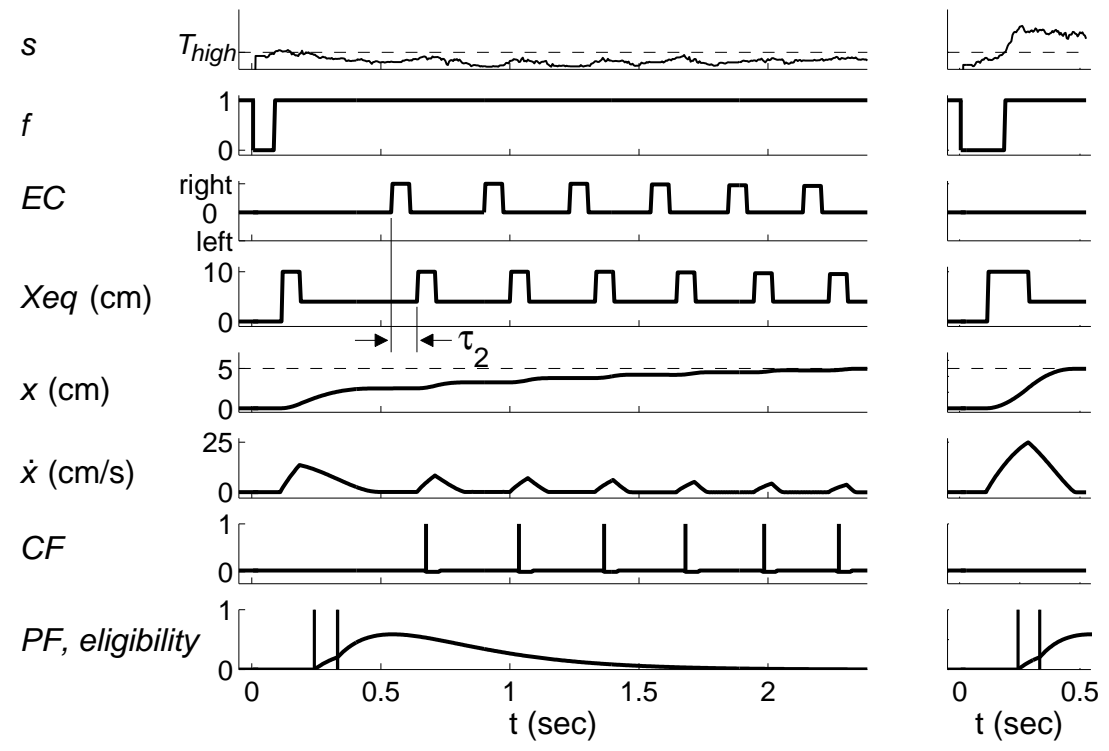

A

$\mathrm{B}$

Figure 4: Single DZ Behavior. The target endpoint, $x_{T}$, was switched from 0 to $5 \mathrm{~cm}$ at time 0 . Shown are the time courses of the DZ's summed input, $s$; activation state, $f$; extracerebellar corrective command, EC; motor command, $x_{e q}$ (after the $100 \mathrm{~ms}$ efferent delay $\tau_{2}$ ); and the position, $x$, and velocity, $\dot{x}$, of the mass for a movement that started at initial position $x_{0}=$ 0 . Plot $\mathrm{CF}$ shows climbing fiber activity, and the bottom plot shows the binary activity of an arbitrarily selected PF together with the eligibility trace of its synapse onto the DZ. (The eligibility trace's amplitude is scaled up to make it easily visible; peak eligibility here is 0.029$) . \quad T_{\text {low }}=0.8$ and $T_{\text {high }}=1$. Panel A: Early in Learning (4 trials). DZ state switched to 1 too soon, which caused the mass to undershoot the target. A sequence of six rightward corrective movements was generated by the extracerebellar system (EC) because all but the last failed to bring the mass close enough to the target. Each corrective movement caused a CF discharge. Each discharge of the selected PF contributed to the eligibility trace because the DZ was in state 1 at these times. The weight of this PF's synapse (not shown) decreased when the CF discharge coincided with nonzero eligibility. Panel B: Late in Learning (1,000 trials). The model consistently produced accurate reaching with fast, smooth movements requiring no corrections (and hence with no CF discharges). To accomplish this, the DZ learned to switch to 1 well before (about $300 \mathrm{~ms}$ ) the endpoint was reached. 


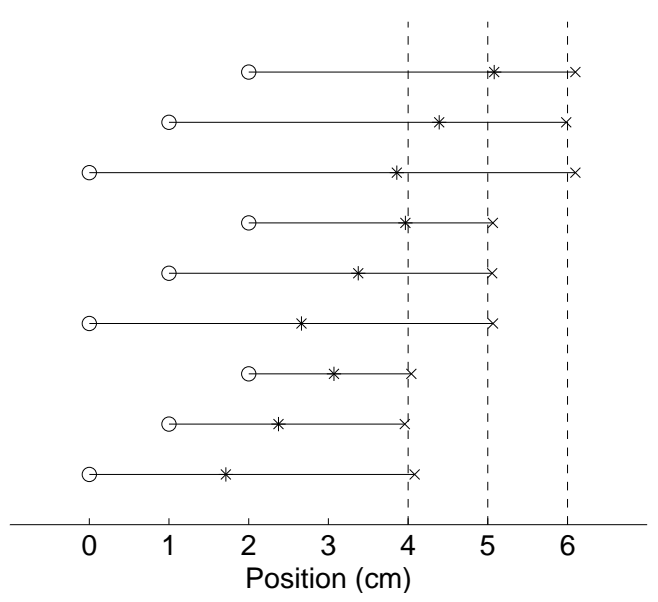

A

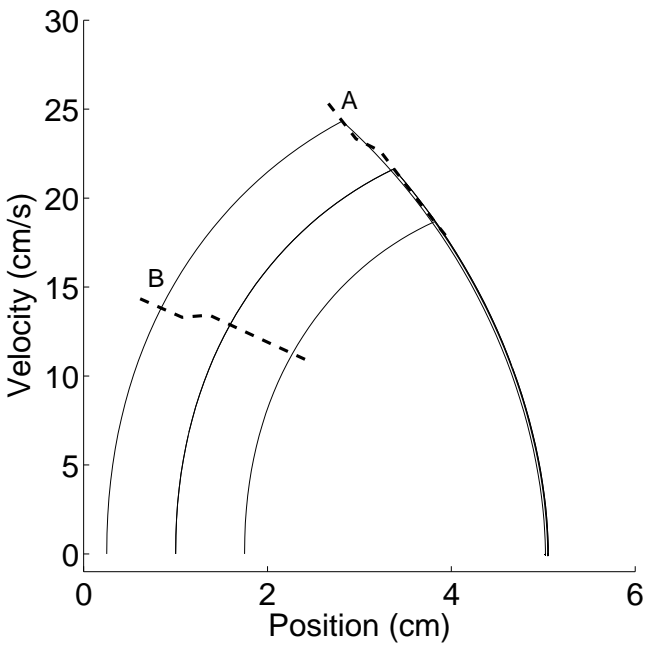

$\mathrm{B}$

Figure 5: Panel A: Paths of a Number of Movements Controlled by a Single Well-Trained DZ. The path of each movement is shown by a horizontal line. The initial position of the mass for each movement is indicated by the circle at the left end of each line, and the target endpoints are indicated by the vertical dashed lines. The asterisk on each path marks the position of the mass when the PC state switched from 0 to 1 . The actual endpoint of the movement is indicated by the ' $x$ ' at the right end of each line. The DZ switches state well before a movement ends. The model used a motor efference delay of 100 ms. Panel B: Switching Curves. Phase-plane portraits of switching curves for target $x_{T}=5 \mathrm{~cm}$ learned by the model. Two switching curves and three example movement trajectories are shown. See text for explanation. 
command input to the spring switches from pulse to step. Clearly, it is positioned correctly to cause the mass to stick close to the desired endpoint for a range of initial conditions. Switching curve B, on the other hand, is the switching curve as it appears before the efferent delay, that is, as seen from the point marked B in Fig. 2A. This curve is crossed $100 \mathrm{~ms}$ before switching curve $\mathrm{A}$ is crossed due to the $100 \mathrm{~ms}$ efferent delay. When the system state crosses this curve, the DZ switches state. One can see, therefore, that the DZ learned to switch $100 \mathrm{~ms}$ before the motor command must switch at the spring itself, appropriately compensating for the $100 \mathrm{~ms}$ latency of the efferent pathway. To do this, the DZ effectively learned to "recognize" the patterns of PF activity that were present at its synapses when the system state crossed switching curve B. It is important to note that due to the various delays in the MF pathways, the recognized PF patterns actually encoded information about the spring-mass state as it was between 15 and $100 \mathrm{~ms}$ earlier.

\subsection{Multiple Dendritic Zones}

We simulated two versions of the model in which the PC consists of 8 DZs. In each case, the PC's activity level at any time is the fraction of its DZs that are in state 1 at that time (see Sec. 3). In one version, each DZ receives input from all of the PFs (uniform model); in the other, each DZ receives input from a separate subfield of 5,000 PFs (subfield model). Fig. 6 shows how the endpoint error decreased with trials for these two variations, as well as for the single DZ model. The uniform model learned significantly faster than the others and reached a smaller final error. We believe this is due to the fact that the uniform model has many more adjustable parameters than the others so that there are many different potential solutions: the algorithm is only constrained to reduce the end-point error, which can be accomplished in many ways. To save computer time, we restricted further simulations to the uniform model, but it is likely that the subfield model would have produced similar results.

Figure 7 illustrates some details of the behavior of the uniform model. Panel $\mathrm{A}$ is analogous to Fig. 4A except that it shows a trial in which there was a single leftward corrective movement instead of multiple rightward corrective movements. Note that the leftward corrective movement did not generate CF discharges but instead slightly depressed CF background rate. Unlike the single DZ case, here the motor command was graded due to the varying contributions of the $8 \mathrm{DZs}$. This variety was due to the differing initial weights of the DZs. Later in learning (1,500 trials, Panel B) one can see 


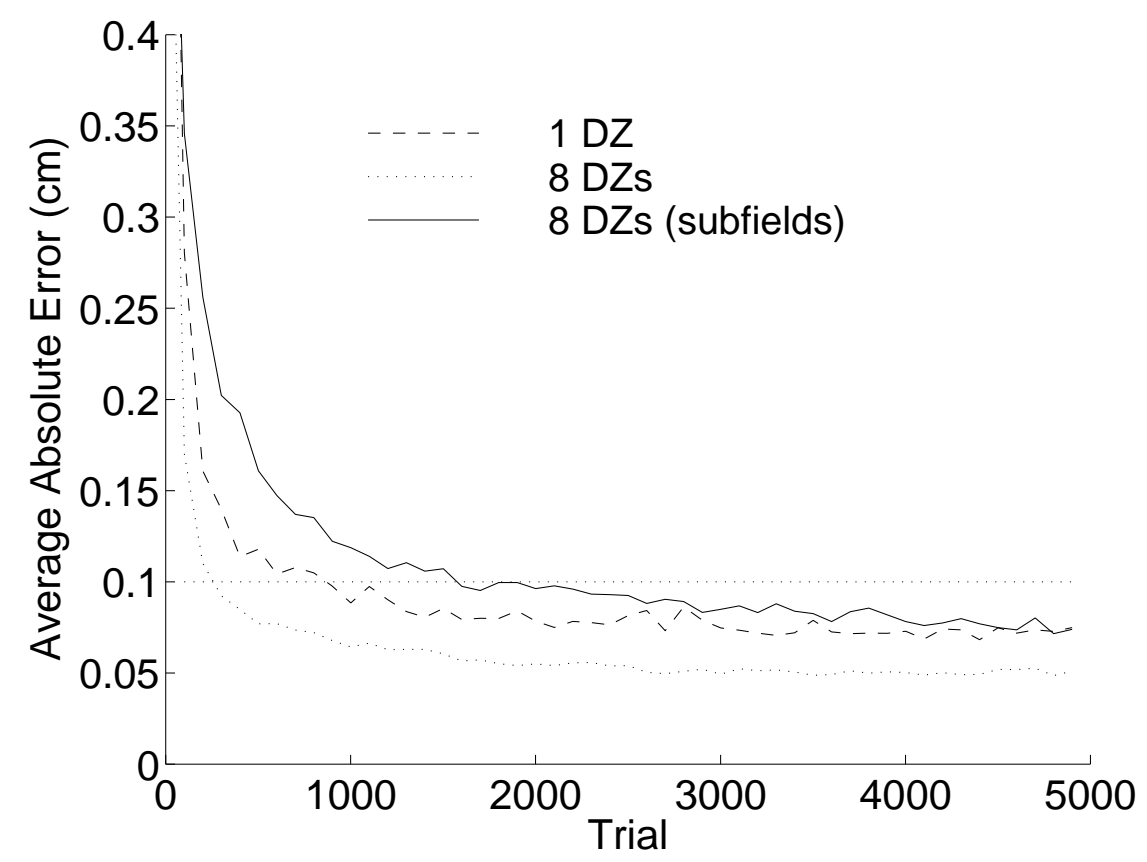

Figure 6: Endpoint Error $\left(\left|x_{e}-x_{T}\right|\right)$ as a Function of Trial Number for MultiDZ Learning. In the uniform model, each DZ receives input from all of the PFs; in the subfield model, each DZ receives input from a separate subfield of 5,000 PFs. Also shown is a plot for the single DZ model. The uniform model learned significantly faster than the others and reached a smaller final error. Each plotted point is an average over a bin of 50 trials of 10 learning runs. The dotted horizontal line represents the minimum threshold above which corrective movements were generated. $T_{\text {low }}=0.8, T_{\text {high }}=1$, and $\tau_{2}=100$ ms. 
that fast, accurate, and smooth movements were accomplished, although the motor command was not a pure pulse.

We also investigated the effects of different levels of hysteresis on the uniform model by fixing $T_{\text {high }}$ to 1 and varying $T_{\text {low }}$, as we did for the single DZ system (Fig. 3B). Unlike the single DZ case, hysteresis had no significant effect on the learning rate of the uniform model. However, we did note that without hysteresis $\left(T_{\text {low }}=1\right.$, Fig. 8$)$, the final motor command was more irregular than it was with hysteresis. This was the result of multiple switching by approximately half of the DZs.

\section{Discussion}

By including realistic conduction delays in afferent and efferent pathways, the model described here allowed the investigation of timing and predictive processes relevant to cerebellar involvement in the control of movement. Moreover, the nonlinearity of the simple motor plant, which is based on muscle mechanical and spinal reflex properties, makes the control problem reflect properties of skeletomotor control better than would a simpler linear plant. While making the control problem more difficult from a conventional control perspective, the nonlinear damping has the advantage of allowing fast movements to be made with little or no oscillation, effectively solving the stability problem, at least for the one-degree-of-freedom positioning task studied here.

Key to the model's ability to perform accurate end-point positioning is its ability to learn predictive control. This is illustrated most clearly in the case of a single dendritic zone (DZ), for which clear switching curves could be derived and related to plant dynamics (Fig. 5B). The model's relative insensitivity to loop delay is due to its predictive use of a rich array of afferent and efference-copy signals. The model does not explicitly predict the motor plant's behavior; that is, it does not use a forward model of the motor plant, a role suggested for the cerebellum by several researchers (Ito 1984; Keeler 1990; Miall et al. 1993). In fact, the model makes no explicit predictions of any kind, if this is taken to mean the creation of representations of future events. Instead, it learns to generate motor commands in a manner that causes desired future behavior. The model is a kind of direct adaptive controller (e.g., Goodwin and Sin 1984), where the term "direct" refers to the lack of a model of the controlled system.

Our model adopts the hypothesis of Marr (1969) and Albus (1971) that the granular layer provides a sparse expansive encoding that increases the ease 


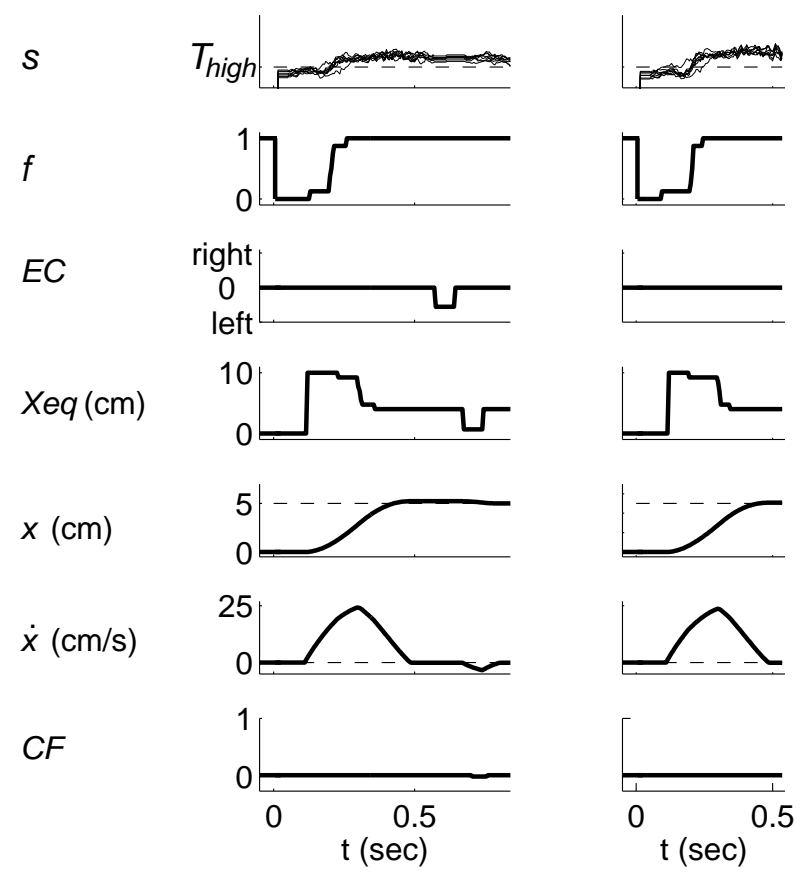

A $\quad$ B

Figure 7: Multiple DZ Behavior. This figure is analogous to Fig. 4 but for a PC consisting of $8 \mathrm{DZs}$, each receiving input from all the PFs (uniform model). The target endpoint, $x_{T}$, was switched from 0 to $5 \mathrm{~cm}$ at time 0 . Shown are the time courses of the 8 DZs' summed inputs, $s$; the PC's activation state, $f$; extracerebellar corrective command, EC; motor command, $x_{e q}$ (after the $100 \mathrm{~ms}$ efferent delay $\tau_{2}$ ); and the position, $x$, and velocity, $\dot{x}$, of the mass for a movement that started at initial position $x_{0}=0$. The bottom plot shows $\mathrm{CF}$ activity. $T_{\text {low }}=0.8$ and $T_{\text {high }}=1$. Panel A: Early in Learning (250 trials). One of the DZs switched back to 1 too late, which caused the mass to slightly overshoot the target. The extracerebellar (EC) system generated a leftward corrective movement, which decreased CF activity below its low background level. Panel B: Late in Learning (1,500 trials). The model consistently produced accurate reaching with fast, smooth movements requiring no corrections. Note that the command is still basically a pulse-step, although it is no longer binary. 


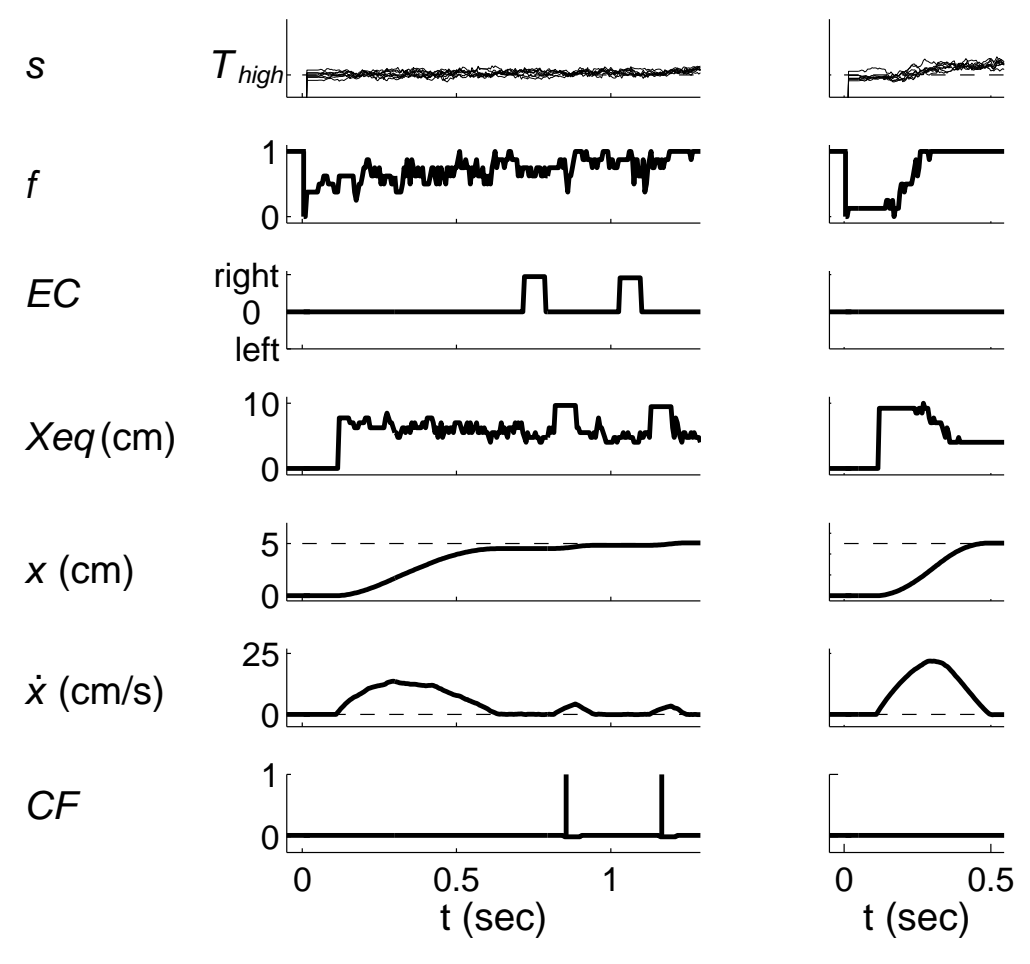

A $\quad$ B

Figure 8: Multiple DZ Behavior Without Hysteresis. This figure is analogous to Fig. 7 except that there was no hysteresis $\left(T_{\text {low }}=1\right)$. Panel A: Early in Learning (175 trials). Panel B: Late in Learning (1,000 trials). The motor command, $x_{e q}$, was more irregular than it was with hysteresis. This was the result of multiple switching by approximately half of the DZs. 
with which a large number of associations can be formed (Buckingham and Willshaw 1992; Tyrrell and Willshaw 1992). We combined this hypothesis with a more realistic representation of movement-related MF signals (Van Kan et al. 1993a). Although the model's use of such a large number of PFs, and hence adjustable parameters (the PF-to-PC synaptic weights), for a such a simple task is a defect from a purely engineering perspective, it is a result of our attempt to faithfully represent what is known about how information is encoded in the MF signals, coupled with our use of a random selection of MF inputs to granule units. Careful design of the latter connection pattern would decrease the number of PFs required. However, simulations show that the current model's behavior degrades if the number of PFs is significantly decreased. We did not investigate the generalization capabilities of the model, which would also be influenced by the input encoding and the number of adjustable parameters.

Experimental data on the effects of CF discharge on PF-to-PC synapses suggest an instructive role for $\mathrm{CF}$ signals, as adopted by the model. There now seems to be good, though not universal, agreement that CF activity, when coupled with other factors, produces long-term depression (LTD) of the action of PF-to-PC synapses (e.g., Crepel 1996 et al.), as postulated by Albus (1971). Less is known about possible long-term potentiation (LTP) at these synapses, which the model also uses, although LTP has been induced in brain slices by stimulating PFs in the absence of CF activity (Sakurai 1987), which is consistent with our model's learning rule.

An essential feature of the model's learning rule is its use of synaptically-local eligibility traces for learning with delayed training information. Eligibility traces are key components of many reinforcement learning systems (e.g., Sutton \& Barto, 1998) as well as models of classical conditioning (Sutton and Barto 1981, 1990; Klopf 1988), where they address the sensitivity of conditioning to the time interval between the conditioned and the unconditioned stimuli and the anticipatory nature of the conditioned response. Eligibility traces play the same role in this model, whose learning mechanism is much like classical conditioning, with corrective movements playing the role of unconditioned responses. ${ }^{5}$ Our model is therefore in accord with the view that general principles of cerebellar-dependent learning may be involved in adaptation of the vestibulo-ocular reflex, classical conditioning of the eyelid response, as well as learning in saccadic eye movements and limb movements (Houk et al. 1996; Raymond et al. 1996). We hypothesize that for reaching,

\footnotetext{
${ }^{5}$ The present model lacks the ability to produce an analog of higher-order conditioning, one of the key features of the above cited classical conditioning models. We know of no studies of the cerebellum's involvement in higher-order classical conditioning.
} 
the role of the cerebellum is to eliminate corrective movements by suitably tuning the initial movement.

Only a few studies of cerebellar plasticity have attempted to manipulate the relative timing of the experimental variables used to elicit LTD. In several studies, LTD occurred only if CF stimulation preceded PF stimulation (Ekerot and Kano 1989; Schreurs and Alkon 1993). Recently, however, Chen and Thompson (1995) demonstrated that delaying CF activation by $250 \mathrm{~ms}$ after a PF volley facilitates the appearance of LTD, suggesting that there may be a cellular mechanism that compensates for the time interval. Schreurs et al. (1996) showed that a form of LTD, which they call pairing-specific longterm depression, results only when $\mathrm{PF}$ stimulation precedes $\mathrm{CF}$ stimulation. Although these studies were motivated by the timing parameters required for classical conditioning of the rabbit nictitating membrane response, their results are relevant to other aspects of motor learning as well. Houk and Alford (1996) presented a model suggesting how intracellular signal transduction mechanisms that mediate LTD could give rise to an eligibility trace. Recent results in which the timing of intracellular signals was controlled photolytically appear to suggest that CF activity should precede PF activity in order to produce LTD (Lev-Ram et al. 1997). However, this conclusion depends critically on the interpretation given to the various intracellular signals. Hopefully, the computational importance of a trace mechanism will stimulate additional cellular studies to explore this critical issue.

The nature of the training information provided by climbing fibers is incompletely understood at present. In oculomotor regions of the cerebellum, CFs are sensitive to retinal 'slip' and thus are well suited to detect errors in the stabilization of visual input. By analogy, one presumes that the somatosensory sensitivity of CFs in limb regions has an analogous error-detection function, although this has been difficult to specify in detail (Fu et al. 1997; Houk et al. 1996; Kitazawa et al. 1998; Simpson et al. 1996). In the present model, we adopted our earlier (Berthier et al. 1993) working hypothesis that CFs detect hypometria by responding to corrective movements in the same direction as the primary movement. This was rationalized from the finding that CFs with directional sensitivity to passive limb movements (units located in the rostral medial accessory olive) are inhibited during self-generated movements (Gellman et al. 1985) but fire when perturbations occur during or at the end of the movement (Andersson and Armstrong, 1987; Horn et al. 1996). We assume that corrective movements occur near the end of inaccurate movements, and that they function like perturbations to fire CFs in a directionally selective manner. 
Reaching movements are known to consist of a primary movement which is often succeeded by one or more secondary movements, the latter being corrective in nature (Prablanc and Martin 1992). Lesion studies have demonstrated the involvement of several neural pathways in the generation of both the primary movements and the corrections (Pettersson et al. 1997). Small corrections do not require vision of the arm and are often made without subject awareness and at shorter latencies than the primary movements (Goodale et al. 1986). These findings suggest the involvement of a simple, automatic mechanism such as the propriospinal network (Alstermark et al. 1984, 1987b). Major corrections, such as reversals in direction, engage the corticospinal system (Georgopoulos et al. 1983). In the present model, we assumed that all corrections following primary movements are made by a simple, extracerebellar process presumed to be mediated by the propriospinal system. This was meant to be a minimalistic assumption; the model could have made use of training information derived from more accurate corrective movements generated, for example, by the corticospinal system. In fact, since training information is derived from the proprioceptive consequences of corrective movements, the model is capable of learning from corrections generated by any system or combination of systems.

We also used the model to experiment with possible computational roles for plateau potentials in PC dendrites (Llinás and Sugimori 1980; Ekerot and Oscarsson 1981; Campbell et al. 1983; Andersson et al. 1984). Our representation of DZs as linear threshold elements with hysteresis allows them to produce abstract analogs of plateau potentials. Hysteresis is sometimes used in two-action control systems to reduce "chattering" caused by repeated crossing of the switching curve. It has the same effect here in making the DZs switch state less frequently, which makes the model's motor commands less erratic. Hysteresis greatly facilitated learning in the single-DZ case, presumably because it prevented chattering in motor commands thereby making them closer to the pulse-step form and thus reducing the amount of learning required. In the multiple-DZ case, hysteresis had little influence on learning, perhaps because the motor commands were relatively smooth without hysteresis since they resulted from the activity of multiple DZs. We did, however, observe increased chatter in the pulse-step command when hysteresis was removed (Fig. 8), suggesting that hysteresis could have a role in facilitating the generation of well-formed motor commands. More study is needed to explore possible computational roles of nonlinear properties of PC dendrites.

Several previous cerebellar models dealing with eye movement are closely related to the model of limb control presented here. Like our model, the model of adaptive control of saccades due to Schweighofer et al. (1996a, b) follows Berthier et al. (1993) and Houk et al. (1990) in making use of corrective 
movements as sources of training information. Schweighofer et al. also use eligibility traces following the classical conditioning models of Sutton and Barto $(1981,1990)$. Unlike the monotonically decaying traces in these models, however, the eligibility traces of Schweighofer et al. reach peaks some time after being initiated. This is in accord with Klopf's (1972) original conception that peak eligibility occurs at the optimal interstimulus interval for learning (see also Klopf 1988). Our model also adopts this type of eligibility trace. The key differences between our model and that of Schweighofer et al. are due to differences in the dynamics of the motor plant and the degree of attention paid to system delays and afferent encoding. Because we are concerned with limb movement, our motor plant has significant inertia, which together with nontrivial delays in various conduction channels, requires significant anticipatory control as illustrated by our simulations. The eye plant of the Schweighofer et al. model lacks significant dynamics (the plant is essentially inertia-less), and it is not apparent that conduction delays are included. The ramp encodings we use for most of the MF signals are also more faithful representations of experimentally-observed MF encodings.

Our model also shares features with the model of predictive smooth pursuit eye movements due to Kettner et al. (1997). Like ours, this model includes mossy fiber inputs with diverse response properties and delays, a granular layer that expansively recodes this input, and a similar learning rule using eligibility traces generated by a second-order linear system. The PCs of that model, however, are continuous elements as opposed to the multistable ones used in the present model (although, as the number of DZs is increased, our model more closely approximates a continuous system). Additionally, training information in the Kettner model is provided by CFs that detect failures of image stabilization (retinal slip) instead of corrective eye movements.

A model of limb movement related to ours is the feedback-error-learning model of Kawato and Gomi $(1992,1993)$ in which the cerebellum learns to act as an inverse dynamic model of the motor plant, being trained by feedback generated from movement caused by an extracerebellar system. This is similar to what we have done in the our model with two exceptions. First, our training information is intermittent feedback from discrete corrective movements, instead of a continuous feedback signal. Second, unlike feedbackerror-learning models, as well as the limb control model of Schweighofer (1995), we do not assume that reference trajectories specifying the complete kinematic details of the desired movement are supplied to the cerebellum by another brain region. Therefore, we do not hypothesize that the cerebellum becomes an inverse dynamic model of the plant in the sense of associating a reference trajectory to appropriate control signals. Target signals in 
our model do not convey this kind of detailed information about the desired trajectory. Instead, through learning, target signals become associated with movements whose kinematic details are determined by the properties of the motor plant. Our model therefore has elements in common with the equilibrium-point hypothesis (Bizzi et al. 1992; Feldman 1966, 1974) in that muscles and spinal reflexes play essential roles in trajectory formation. Unlike that hypothesis, however, movement endpoints are generally not equilibrium positions.

The model presented in this paper has a number of limitations. It lacks representations of many of the components of the full APG model on which it is based. In that model, movement would be the result of the combined effects of the elemental commands of a number of cerebellar APG modules that operate simultaneously. Here, we described only a single module consisting of a single PC, and included no explicit representation of premotor circuits. Because the model presented here consists of a single PC controlling a single agonist actuator, it does not illustrate critical features of the full model. It does not show, for example, that during a movement most PCs would have to increase activity to inhibit muscle synergies that should not fully participate in the movement. In the model presented here, the single PC always has to decrease activity to generate a motor command. Our model also suggests that after learning, the extracerebellar source of corrective movements no longer plays a role in limb movement. While this is consistent with the feedbackerror-learning model, it is at variance with models of saccade generation in which cerebellar control augments, rather than replaces, the control provided by the brainstem burst generator (Dean 1995; Arai et al. 1994; Optican 1995). Our model does not adopt this approach because much less is known about the propriospinal network than is known about the brainstem pulse generator. However, this would be worthwhile to pursue in future research.

Finally, nothing in the present paper suggests how the model presented here might extend to more complex control problems involving multi-degree-offreedom limbs. One of the objectives of the full APG model is to explore how the collective behavior of multiple APG modules can accomplish pulsestep control of a more complex motor plant without resorting to pre-planned reference trajectories. Our research is continuing in this direction (Fagg et al. 1997a, b).

\section{Acknowledgments}

This work was supported by NIH 1-50 MH 48185. We thank Jay Buckingham for his contributions to an earlier version of this model, and Sascha 
Engelbrecht for helpful comments.

\section{References}

[Albus, 1971] Albus, J. S. (1971). A theory of cerebellar function. Mathematical Biosciences, 10:25-61.

[Alstermark et al., 1984] Alstermark, B., Eide, E., Górska, T., Lundberg, A., and Pettersson, L. G. (1984). Visually guided switching of forelimb target reaching in cats. Acta. Physiol. Scand., 120:151-153.

[Alstermark et al., 1987a] Alstermark, B., Lundberg, A., Pinter, M. J., and Sadaki, S. (1987a). Long C3-C5 propriospinal neurones in the cat. Brain Research, 404:382-388.

[Alstermark et al., 1987b] Alstermark, B., T. Górska, A. L., Pettersson, L. G., and Walkowska, M. (1987b). Effect of different spinal cord lesions on visually guided switching of target-reaching in cats. Neuroscience Research, 5:63-67.

[Andersson and Armstrong, 1987] Andersson, G. and Armstrong, D. M. (1987). Complex spikes in purkinje cells in the lateral vermis (b zone) of the cat cerebellum during locomotion. Journal of Physiology, London, $385: 107-134$.

[Andersson et al., 1984] Andersson, G., Campbell, N. C., Ekerot, C. F., Hesslow, G., and Oscarsson, O. (1984). Integration of mossy fiber and climbing fiber inputs to Purkinje cells. Experimental Brain Research (Suppl), 9:145-150.

[Arai et al., 1994] Arai, K., Keller, E. L., and Edelman, J. A. (1994). Twodimentional neural network model of the primate saccadic system. Neural Networks, 7:1115-1135.

[Barto et al., 1996] Barto, A. G., Buckingham, J. T., and Houk, J. C. (1996). A predictive switching model of cerebellar movement control. In Touretzky, D. S., Mozer, M. C., and Hasselmo, M. E., editors, Advances in Neural Information Processing Systems: Proceedings of the 1995 Conference, pages 138-144, Cambridge, MA. MIT Press.

[Benson et al., 1987] Benson, M. W., Bree, G. M., Kinahan, P. E., and Hoffmann, G. W. (1987). A teachable neural network based on an unorthodox neuron. Physica D, 22:233-246. 
[Berthier et al., 1993] Berthier, N. E., Singh, S. P., Barto, A. G., and Houk, J. C. (1993). Distributed representations of limb motor programs in arrays of adjustable pattern generators. Journal of Cognitive Neuroscience, 5:5678 .

[Bizzi et al., 1992] Bizzi, E., Hogan, N., Mussa-Ivaldi, F. A., and Gister, S. (1992). Does the nervous system use equilibrium-point control to guide single and multiple joint movements? Behavioral and Brain Sciences, 15:603-613.

[Buckingham et al., 1995] Buckingham, J. T., Barto, A. G., and Houk, J. C. (1995). Adaptive predictive control with a cerebellar model. In Proceedings of the 1995 World Congress on Neural Networks, pages 373-380, Mahwah, NJ. Lawrence Erlbaum Associates.

[Buckingham and Willshaw, 1992] Buckingham, J. T. and Willshaw, D. (1992). A note on the storage capacity of the associative net. Network, $3(4)$.

[Campbell et al., 1983] Campbell, N. C., Ekerot, C. F., Hesslow, G., and Oscarsson, O. (1983). Dendritic plateau potentials evoked in Purkinje cells by parallel fibre volleys in the cat. Journal of Physiology (Lond), 340:209-223.

[Chen and Thompson, 1995] Chen, C. and Thompson, R. F. (1995). Temporal specificity of long-term depression in parallel fiber - Purkinje synapses in rat cerebellar slice. Learning and Memory, 2:185-198.

[Crepel et al., 1996] Crepel, F., Hemart, N., Jaillard, D., and Daniel, H. (1996). Cellular mechanisms of long-term depression in the cerebellum. Behavioral and Brain Sciences, 19(3):347.

[Dean, 1995] Dean, P. (1995). Modelling the role of the cerebellar fastigial nuclei in producing accurate saccades: the importance of burst timing. Neuroscience, 68:1059-1077.

[Ekerot and Kano, 1989] Ekerot, C. F. and Kano, M. (1989). Stimulation parameters influencing climbing fiber induced long-term depression of parallel fiber synapses. Neuroscience Research, 6:264-268.

[Ekerot and Oscarsson, 1981] Ekerot, C. F. and Oscarsson, O. (1981). Prolonged depolarization elicited in Purkinje cell dendrites by climbing fiber impulses in the cat. Journal of Physiology (Lond), 318:207-221. 
[Fagg et al., 1997a] Fagg, A. H., Sitkoff, N., Barto, A. G., and Houk, J. C. (1997a). Cerebellar learning for control of a two-link arm in muscle space. In Proceedings of the IEEE Conference on Robotics and Automation, pages 2638-2644.

[Fagg et al., 1997b] Fagg, A. H., Sitkoff, N., Barto, A. G., and Houk, J. C. (1997b). A model of cerebellar learning for control of arm movements using muscle synergies. In Proceedings of the IEEE Symposium on Computational Intelligence in Robotics and Automation, pages 6-12.

[Feldman, 1966] Feldman, A. (1966). Functional tuning of the nervous system with control of movement or maintenance of a steady posture. II Controllable parameters of the muscle. Biophysics, 11:565-578.

[Feldman, 1974] Feldman, A. (1974). Change in length of the muscle as a consequence of the shift in equilibrium in the muscle-load system. Biophysics, 19:544-548.

[Fu et al., 1997] Fu, Q.-G., Mason, C. R., Flament, D., Coltz, J. D., and Ebner, T. J. (1997). Movement kinematics encoded in complex spike discharge of primate cerebellar purkinje cells. NeuroReport, 8:523-529.

[Gellman et al., 1983] Gellman, R., Gibson, A. R., and Houk, J. C. (1983). Somatosensory properties of the inferior olive of the cat. Journal of Comparative Neurology, 215:228-243.

[Gellman et al., 1985] Gellman, R., Gibson, A. R., and Houk, J. C. (1985). Inferior olivary neurons in the awake cat: Detection of contact and passive body displacement. Journal of Neurophysiology, 54:40-60.

[Georgopoulos et al., 1983] Georgopoulos, A. P., Kalaska, J. F., Caminiti, R., and Massey, J. T. (1983). Interruption of motor cortical discharge subserving aimed arm movements. Experimental Brain Research, 49:327340 .

[Ghez, 1979] Ghez, C. (1979). Contributions of central programs to rapid limb movement in the cat. In Asanuma, H. and Wilson, V. J., editors, Integration in the Nervous System, pages 305-320. Igaku-Shoin, Tokyo.

[Ghez et al., 1990] Ghez, C., Gordon, J., Ghilardi, M. F., Christakos, C. N., and Cooper, S. E. (1990). Roles of proprioceptive input in the programming of arm trajectories. In The Brain: Cold Spring Harbor Symp. Quant. Biol.; Volume LV, pages 837-847. Cold Spring Harbor Laboratory Press. 
[Ghez and Martin, 1982] Ghez, C. and Martin, J. H. (1982). The control of rapid limb movment in the cat. III. Agonist-antagonist coupling. Experimental Brain Research, 45:115-125.

[Gielen and Houk, 1984] Gielen, C. C. A. M. and Houk, J. C. (1984). Nonlinear viscosity of human wrist. Journal of Neurophysiology, 52:553-569.

[Gielen and Houk, 1987] Gielen, C. C. A. M. and Houk, J. C. (1987). A model of the motor servo: Incorporating nonlinear spindle receptor and muscle mechanical properties. Biological Cybernetics, 57:217-231.

[Goodale et al., 1986] Goodale, M. A., Pélisson, D., and Prablanc, C. (1986). Large adjustments in visually guided reaching do not depend on vision of the hand or perception of target displacement. Nature, 320:748-750.

[Goodwin and Sin, 1984] Goodwin, G. C. and Sin, K. S. (1984). Adaptive Filtering Prediction and Control. Prentice-Hall, Englewood Cliffs, N.J.

[Gutman, 1991] Gutman, A. M. (1991). Bistability of dendrites. International Journal of Neural Systems, 1:291-304.

[Gutman, 1994] Gutman, A. M. (1994). Gelfand-Tsetlin principle of minimal afferentation and bistability of dendrites. International Journal of Neural Systems, 5:83-86.

[Hoffman, 1986] Hoffman, G. W. (1986). A neural network model based on the analogy with the immune system. Journal of Theoretical Biology, 122:33-67.

[Horn et al., 1996] Horn, K. M., Van Kan, P. L. D., and Gibson, A. R. (1996). Reduction of rostral dorsal acessory olive responses during reaching. Journal of Neurophysiology, 76:4140-4151.

[Houk, 1989] Houk, J. C. (1989). Cooperative control of limb movements by the motor cortex. In Cotterill, R. M. J., editor, Models of Brain Function, pages 309-325. Cambridge University Press, Cambridge.

[Houk and Alford, 1996] Houk, J. C. and Alford, S. (1996). Computational significance of the cellular mechanisms for synaptic plasticity in Purkinje cells. Behavioral and Brain Sciences, 19(3):457.

[Houk and Barto, 1992] Houk, J. C. and Barto, A. G. (1992). Distributed sensorimotor learning. In Stelmach, G. E. and Requin, J., editors, Tutorials in Motor Behavior II, pages 71-100. Elsevier Science Publishers B. V., Amsterdam, The Netherlands. 
[Houk et al., 1996] Houk, J. C., Buckingham, J. T., and Barto, A. G. (1996). Models of the cerebellum and motor learning. Behavioral and Brain Sciences, 19:368-383.

[Houk et al., 1992] Houk, J. C., Galiana, H. L., and Guitton, D. (1992). Cooperative control of gaze by the superior colliculus, brainstem and cerebellum. In Stelmach, G. E. and Requin, J., editors, Tutorials in Motor Behavior II, pages 443-474. Elsevier Science Publishers B. V., Amsterdam, The Netherlands.

[Houk et al., 1993] Houk, J. C., Keifer, J., and Barto, A. G. (1993). Distributed motor commands in the limb premotor network. Trends in Neuroscience, 16:27-33.

[Houk and Rymer, 1981] Houk, J. C. and Rymer, W. Z. (1981). Neural control of muscle length and tension. In Brooks, V. B., editor, Handbook of Physiology: Sec. 1: Vol. 2. Motor Control, pages 247-323. American Physiological Society, Bethesda, MD.

[Houk et al., 1990] Houk, J. C., Singh, S. P., Fisher, C., and Barto, A. G. (1990). An adaptive network inspired by the anatomy and physiology of the cerebellum. In Miller, T., Sutton, R. S., and Werbos, P. J., editors, Neural Networks for Control, pages 301-348. MIT Press, Cambridge, MA.

[Houk and Wise, 1995] Houk, J. C. and Wise, S. P. (1995). Distributed modular architectures linking basal ganglia, cerebellum and cerebral cortex: Their role in planning and controlling action. Cerebral Cortex, 5:95-110.

[Ito, 1984] Ito, M. (1984). The Cerebellum and Neural Control. Raven Press, New York.

[Kawato and Gomi, 1992] Kawato, M. and Gomi, H. (1992). A computational model of four regions of the cerebellum based on feedback-error learning. Biological Cybernetics, 68:95-103.

[Kawato and Gomi, 1993] Kawato, M. and Gomi, H. (1993). Feedbackerror-learning model of cerebellar motor control. In Mano, N., Hamada, I., and DeLong, M. R., editors, Role of the Cerebellum and Basal Ganglia in Voluntary Movement, pages 51-61. Elsevier Science Pub. B. V.

[Keeler, 1990] Keeler, J. D. (1990). A dynamical system view of cerebellar function. Physica D, 42:396-410.

[Keller and Robinson, 1971] Keller, E. L. and Robinson, D. A. (1971). Absence of stretch reflex in extraocular muscles of the monkey. Journal of Neurophysiology, 34:908-919. 
[Kettner et al., 1997] Kettner, R. E., Mahamud, S., Leung, H.-C., Barto, A. G., Houk, J. C., Peterson, B. W., and Sitkoff, N. (1997). Prediction of complex two-dimensional trajectories by the eye and by a cerebellar model of smooth eye movement. Journal of Neurophysiology, 77:2115-2130.

[Kiehn, 1991] Kiehn, O. (1991). Plateau potentials and active integration in the 'final common pathway' for motor behavior. Trends in Neuroscience, 14:68-73.

[Kitazawa et al., 1998] Kitazawa, S., Kimura, T., and Yin, P.-B. (1998). Cerebellar complex spikes encode both destinations and errors in arm movements. Nature, 392:494-497.

[Klopf, 1972] Klopf, A. H. (1972). Brain function and adaptive systems-A heterostatic theory. Technical Report AFCRL-72-0164, Air Force Cambridge Research Laboratories, Bedford, MA. A summary appears in Proceedings of the International Conference on Systems, Man, and Cybernetics, 1974, IEEE Systems, Man, and Cybernetics Society, Dallas, TX.

[Klopf, 1982] Klopf, A. H. (1982). The Hedonistic Neuron: A Theory of Memory, Learning, and Intelligence. Hemisphere, Washington, D.C.

[Klopf, 1988] Klopf, A. H. (1988). A neuronal model of classical conditioning. Psychobiology, 16:85-125.

[Lev-Ram et al., 1997] Lev-Ram, V., Jiang, T., Wood, J., Lawrence, D. S., and Tsien, R. Y. (1997). Synergies and coincidence requirements between NO, cGMP, and ca2+ in the induction of cerebellar long-term depression. Neuron, 18:1025-1038.

[Llinás and Sugimori, 1980] Llinás, R. and Sugimori, M. (1980). Electrophysiological properties of in vitro Purkinje cell dendrites in mammalian cerebellar slices. Journal of Physiology (London), 305:197-213.

[Marr, 1969] Marr, D. (1969). A theory of cerebellar cortex. Journal of Physiology (Lond), 202:437-470.

[Miall et al., 1993] Miall, R. C., Weir, D. J., Wolpert, D. M., and Stein, J. F. (1993). Is the cerebellum a smith predictor? Journal of Motor Behavior, 25:203-216.

[Optican, 1995] Optican, L. M. (1995). A field theory of saccade generation: Temporal-to-spatial transform in the superior colliculus. Vision Research, 35:3313-3320. 
[Optican and Robinson, 1980] Optican, L. M. and Robinson, D. A. (1980). Cerebellar-dependent adaptive control of primate saccadic system. Journal of Neurophysiology, 44:1058-1076.

[Pettersson et al., 1997] Pettersson, L.-G., Lundberg, A., Alstermark, B., Isa, T., and Tantisira, B. (1997). Effect of spinal cord lesions on forelimb target-reaching and on visually guided switching of target-reaching in the cat. Neuroscience Research, 29:241-256.

[Prablanc and Martin, 1992] Prablanc, C. and Martin, O. (1992). Automatic control during hand reaching at undetected two-dimensional target displacements. Journal of Neurophysiology, 67:455-469.

[Raymond et al., 1996] Raymond, J. L., Lisberger, S. G., and Mauk, M. D. (1996). The cerebellum: A neuronal learning machine? Science, 272(5265):1126-1131.

[Robinson, 1975] Robinson, D. A. (1975). Oculomotor control signals. In Lennerstrand, G. and Bach-y-rita, P., editors, Basic Mechanisms of Ocular Motility and their Clinical Implications, pages 337-374. Pergamon Press, Oxford.

[Sakurai, 1987] Sakurai, M. (1987). Synaptic modification of parallel fibrePurkinje cell transmission in in vitro guinea-pig cerebellar slices. Journal of Physiology (Lond), 394:463-480.

[Schreurs and Alkon, 1993] Schreurs, B. G. and Alkon, D. L. (1993). Rabbit cerebellar slice analysis of long-term depression and its role in classical conditioning. Brain Research, 631:235-240.

[Schreurs et al., 1996] Schreurs, B. G., Oh, M. M., and Alkon, D. L. (1996). Pairing-specific long-term depression of Purkinje cell excitatory postsynaptic potentials results from a classical conditioning procedure in the rabbit cerebellar slice. Journal of Physiology, 75:1051-1060.

[Schweighofer, 1995] Schweighofer, N. (1995). Computational Models of the Cerebellum in the Adaptive Control of Movements. PhD thesis, University of Southern California, Los Angeles, CA.

[Schweighofer et al., 1996a] Schweighofer, N., Arbib, M. A., and Dominey, P. F. (1996a). A model of the cerebelllum in adaptive control of saccadic gain I. The model and its biological substrate. Biological Cybernetics, $75: 19-28$. 
[Schweighofer et al., 1996b] Schweighofer, N., Arbib, M. A., and Dominey, P. F. (1996b). A model of the cerebelllum in adaptive control of saccadic gain II. Simulation results. Biological Cybernetics, 75:29-36.

[Simpson et al., 1996] Simpson, J. I., Wylie, D. R., and de Zeeuw, C. I. (1996). On climbing fiber signals and their consequence(s). Behavioral and Brain Sciences, 19:384-398.

[Sinkjær et al., 1990] Sinkjær, T., Wu, C. H., Barto, A. G., and Houk, J. C. (1990). Cerebellar control of endpoint position - A simulation model. In Proceedings of the 1990 International Joint Conference on Neural Networks, pages II-705-710, San Diego, CA.

[Sutton and Barto, 1981] Sutton, R. S. and Barto, A. G. (1981). Toward a modern theory of adaptive networks: Expectation and prediction. Psychological Review, 88:135-170.

[Sutton and Barto, 1990] Sutton, R. S. and Barto, A. G. (1990). Timederivative models of Pavlovian reinforcement. In Gabriel, M. and Moore, J., editors, Learning and Computational Neuroscience: Foundations of Adaptive Networks, pages 497-537. MIT Press, Cambridge, MA.

[Sutton and Barto, 1998] Sutton, R. S. and Barto, A. G. (1998). Reinforcement Learning: An Introduction. MIT Press, Cambridge, MA.

[Tyrrell and Willshaw, 1992] Tyrrell, T. and Willshaw, D. J. (1992). Cerebellar cortex: Its simulation and the relevance of Marr's theory. Proceedings of the Royal Society of London B, 336:239-257.

[Van Kan et al., 1993a] Van Kan, P. L. E., Gibson, A. R., and Houk, J. C. (1993a). Movement-related inputs to intermediate cerebellum of the monkey. Journal of Neurophysiology, 69:74-94.

[Van Kan et al., 1993b] Van Kan, P. L. E., Houk, J. C., and Gibson, A. R. (1993b). Output organization of interemediate cerebellum of the monkey. Journal of Neurophysiology, 69:57-73.

[Wang and Ross, 1990] Wang, L. and Ross, J. (1990). Synchronous neural networks of nonlinear threshold elements with hysteresis. Proceedings of the National Academy of Science USA, 87:988-992.

[Wu et al., 1990] Wu, C. H., Houk, J. C., Young, K. Y., and Miller, L. E. (1990). Nonlinear damping of limb motion. In Winters, J. M. and Woo, S. L.-Y., editors, Multiple Muscle Systems: Biomechanics and Movement Organization, pages 214-235. Springer-Verlag, New York. 
[Yuen et al., 1995] Yuen, G. L., Hockberger, P. E., and Houk, J. C. (1995). Bistability in cerebellar Purkinje cell dendrites modelled with highthreshold calcium and delayed-rectifier potassium channels. Biological Cybernetics, 73:375-388. 